\title{
Lesson of the month 2: Against all odds
}

\author{
Author: Ami Schattner ${ }^{\mathrm{A}}$
}

When a patient who suffers from a serious underlying disease with an ever-present potential for recurrence or progression deteriorates, other alternative diagnoses (even treatable ones) are frequently not considered. However, these patients are often immunosuppressed, which makes them susceptible to reactivation of latent viral or tuberculosis infections. Three brief cases are presented to stress the importance of looking for unsuspected, treatable herpes virus infections in these settings and the relevant cognitive aspects of misdiagnosis are discussed.

KEYWORDS: Cancer, lymphoma, ulcerative colitis, immunosuppression, herpes virus, tuberculosis, differential diagnosis

\section{Introduction}

When a patient who suffers from a serious underlying disease with an ever-present potential for recurrence or progression deteriorates, other alternative diagnoses may not be seriously considered. Three brief cases are presented here to stress the importance of an alternative outlook and suggest some principles that may help prevent diagnostic errors and identify rare but treatable infectious causes to which these patients are also susceptible.

\section{Case histories}

\section{Case 1}

A young woman with flare-up of her ulcerative colitis (UC), refractory to all treatment, was scheduled for total colectomy. A last minute presentation based on a Medline search ('ulcerative colitis AND complications'), given by an intern, uncovered a report of cytomegalovirus (CMV) infection masquerading as an ulcerative colitis flare-up. When subsequently superimposed CMV colitis was actively sought, it was immediately confirmed by the finding of typical inclusion bodies in biopsies and, later, viral cultures. ${ }^{1}$ Susceptibility may be increased by either the disease, ${ }^{2,3}$ its treatment, or both. The patient responded completely to ganciclovir.

\section{Case 2}

A 67-year-old man with longstanding small lymphocytic lymphoma (SLL) stage IV had recurrent disease after 10 years

Author: ${ }^{A}$ professor of medicine, Hebrew University and Hadassah Medical School, Jerusalem, Israel of remission, which responded to treatment. He then became symptomatic and developed a growing cervical lymph node mass. Transformation of SLL into a more malignant form was strongly suspected. However, several atypical features, as well as a literature search, supported the possibility of virus reactivation associated with the patient's rituximab treatment. Rituximab causes B-cell depletion and has been convincingly associated with an increased risk of infections in general, and viral infections in particular. ${ }^{4,5}$ Immunohistochemical analysis of a biopsy using anti-herpes simplex virus (HSV) antibodies led to the discovery of HSV lymphadenitis. The mass rapidly decreased on valcyclovir treatment. ${ }^{6}$

\section{Case 3}

A 68-year-old woman had a remote history of treated breast cancer (2001) and a more recently discovered and treated stage IIIB small cell lung cancer (SCLC) (2010). She presented in 2015 with recent onset of headaches and 4 days of apathy. On admission she was afebrile but stuporous, and grand mal seizures developed soon after. Although computed tomography imaging was non-contributory, central nervous system recurrence was considered practically certain since lung and breast carcinomas are among the most common primary tumours responsible for brain metastases. ${ }^{7}$ Magnetic resonance imaging (MRI) was ordered. However, on the third hospital day she developed fever and a grouped vesicular eruption around her eye. The cerebrospinal fluid showed pleocytosis, elevated protein concentration and polymerase chain reaction was positive for varicella-zoster virus DNA. The electroencephalogram was consistent with encephalitis. MRI was normal. She completely responded to intravenous acyclovir.

\section{Discussion}

Against all odds, the patient with UC and intractable diarrhoea did not have a refractory disease (estimated to occur in up to $40 \%$ of patients with UC) but had uncommon CMV colitis instead; the patient with SLL and increasing neck mass did not have Richter's transformation (estimated to occur in up to $13 \%$ of SLL patients) but a rare HSV lymphadenitis; and the patient with a history of both SCLC and breast cancer who developed seizures did not have cerebral metastases (estimated to occur in over $50 \%$ of patients at 5 years) but uncommon VZV encephalitis.

As our patients' chart notes reveal, highly experienced physicians in charge of these patients were certain of what turned out to be a misdiagnosis. Cognitive errors played a 
central role in the diagnostic delay in the patients, as is usually found. ${ }^{8}$ Fortunately, it lasted only a few days. Clinicians at all levels very often depend on an unconscious, rapid ('augenblik') 'pattern recognition' for diagnosis, which is dependent on prior examples. Thus, the propensity for error in rare diagnoses, which require careful analytical reasoning, is quite high. Moreover, this type of reasoning depends on heuristics (mental shortcuts) that are very prone to distraction by contextual factors (such as the overriding illness itself) and an array of biases (such as availability and framing biases). ${ }^{9}$ Both are hard to avoid and deserve better recognition. ${ }^{10}$

The underlying disease and its treatment may mediate immunosuppression - significant enough for a serious latent infection to emerge, albeit rarely. This applies, in particular, to latent herpes virus infections as well as tuberculosis. ${ }^{3,10}$

I suggest five practical principles to increase clinicians' awareness of this contingency and prevent diagnostic errors:

1 The same factors that predispose the patient to a possible deterioration of the basic disease or its immunosuppressive treatment may underlie activation of a latent serious infection, viral or otherwise. ${ }^{11}$

2 This activation of a latent infection may mimic a major facet of the underlying disease and seem prognostically doomed. Nevertheless, these infections are highly treatable.

3 Rare diagnostic possibilities can seldom be instinctively recalled. A database search for a full differential diagnosis should be performed routinely.

4 Although the chance of identifying a treatable serious infection is slim, it constitutes a realistic possibility - a $100 \%$ probability for the involved single patient.

5 'Never say never' - always insist on obtaining a tissue diagnosis (or body fluid) and ask a specific question.

\section{Conflicts of interest}

The author declares no conflicts of interest.

\section{Acknowledgements}

Written informed consent was obtained from the patients to publish the clinical details included in this article.

\section{References}

1 Schattner A, Gabovich N, Lifschiz A, Becker S. Medline solution. Lancet 1999; 353:462.

2 Soderberg-Naucler C, Fish KN, Nelson JA. Interferon-g and tumor necrosis factor-a specifically induce formation of cytomegaloviruspermissive monocyte-derived macrophages that are refractory to the antiviral activity of these cytokines. J Clin Invest 1997;100:3154-63.

3 Nguyen M, Bradford K, Zhang X, Shih DQ. Cytomegalovirus Reactivation in Ulcerative Colitis Patients. Ulcers 2011;2011:282507.

4 Aksoy S, Harputluoglu H, Kilickap S et al. Rituximab-related viral infections in lymphoma patients. Leuk Lymphoma 2007;48:1307-12.

5 Lanini S, Molloy AC, Prentice AG et al. Infections in patients taking rituximab for hematologic malignancies: two-year cohort study. BMC Infect Dis 2013;13:317.

6 Klepfish A, Vaknine H, Schattner A. Never say never: unexpected herpes lymphadenitis. Lancet 2014;384:1640.

7 Tsao MN, Lloyd N, Wong RK et al. Whole brain radiotherapy for the treatment of newly diagnosed multiple brain metastases. Cochrane Database Syst Rev 2012;(4);CD003869.

8 Graber ML, Franklin N, Gordon R. Diagnostic errors in internal medicine. Arch Intern Med 2005;165:1493-9.

9 Croskerry P, Singhal G, Mamede S. Cognitive debiasing 1: origins of bias and theory of debiasing. BMJ Qual Saf 2013;22:ii58-ii64.

10 Schattner A. Researching and preventing diagnostic errors: chasing patient safety from a different angle. QJM 2016;109:293-4.

11 Lakhanpal A, Arfon S, McKeon DJ. So, they thought it was all over. BMJ Case Rep 2011;2;2011.pii:bcr1120103493.

Address for correspondence: Dr A Schattner, Faculty of Medicine, Hebrew University and Hadassah Medical School, Ein Kerem, Jerusalem 91120, Israel.

Email: amischatt@gmail.com 